\title{
Activity of rheumatoid arthritis correlates with oral inflammatory burden
}

\section{Äyräväinen, Leena}

2018-09

Äyräväinen , L, Heikkinen, A M , Kuuliala , A, Ahola , K, Koivuniemi , R , Peltola , J , Suomalainen, A, Moilanen, E , Hämäläinen, M , Laasonen , L, Meurman , J H \& Leirisalo-Repo , M 2018 , ' Activity of rheumatoid arthritis correlates with oral inflammatory burden ' , Rheumatology International , vol. 38 , no. 9 , pp. 1661-1669 . https://doi.org/10.1007/s00296-018-4108-z

http://hdl.handle.net/10138/305117

https://doi.org/10.1007/s00296-018-4108-z

publishedVersion

Downloaded from Helda, University of Helsinki institutional repository.

This is an electronic reprint of the original article.

This reprint may differ from the original in pagination and typographic detail.

Please cite the original version. 


\title{
Activity of rheumatoid arthritis correlates with oral inflammatory burden
}

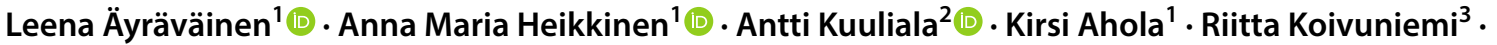 \\ Jaakko Peltola ${ }^{4}$. Anni Suomalainen ${ }^{4}$. Eeva Moilanen ${ }^{5} \odot$ - Mari Hämäläinen ${ }^{5} \odot$ - Leena Laasonen ${ }^{4}$. \\ Jukka H. Meurman ${ }^{1}$ (D) - Marjatta Leirisalo-Repo ${ }^{3}$
}

Received: 14 May 2018 / Accepted: 16 July 2018 / Published online: 24 July 2018

(c) Springer-Verlag GmbH Germany, part of Springer Nature 2018

\begin{abstract}
To study oral health in patients with rheumatoid arthritis (RA) with emphasis on disease activity and treatment of RA. In this prospective cohort study 81 RA patients [53 early untreated RA (EURA) and 28 chronic RA (CRA) patients with inadequate response to synthetic disease modifying antirheumatic drugs (DMARDs)], underwent rheumatological [Disease Activity Score (28-joint) DAS28] and dental examinations [Total Dental Index (TDI), Decayed Missing Filled Teeth (DMFT) and Decayed Missing Filled Surfaces (DMFS)]. For controls, 43 volunteers were examined. After the examinations, EURA patients started treatment with synthetic DMARDs, oral and intra-articular glucocorticoids. CRA patients were candidates for biological DMARDs. The patients were re-examined mean 16 months later. Results were analyzed with descriptive statistics and logistic regression. TDI was higher in both RA groups at baseline compared to controls [EURA: 2 (2-3); CRA: $2(1-3)$; controls $1(1-3), p=0.045]$. DMFT $\left[r_{\mathrm{s}} 0.561(p=0.002)\right]$ and DMFS $\left[r_{\mathrm{s}} 0.581(p=0.001)\right]$ associated with DAS28 at baseline in CRA patients. After follow-up, DAS28 associated positively with DMFT $\left[r_{\mathrm{s}} 0.384(p=0.016)\right]$ and DMFS $\left[r_{\mathrm{s}} 0.334(p=0.038)\right]$ in EURA patients; as well as in CRA patients DMFT $\left[r_{\mathrm{s}} 0.672(p=0.001)\right]$, DMFS $\left[r_{\mathrm{s}} 0.650\right.$ $(p=0.001)]$. RA patients already in the early phase of the disease had poorer oral health compared to controls. The caries indices associated with the activity of RA in both patient groups. Oral status may thus contribute to the development and further relate to the activity of RA.
\end{abstract}

Keywords Arthritis, rheumatoid · Oral health · Antirheumatic agents

Leena Äyräväinen

leena.ayravainen@helsinki.fi

Anna Maria Heikkinen

anna.m.heikkinen@helsinki.fi

Antti Kuuliala

antti.kuuliala@helsinki.fi

Kirsi Ahola

kirsi.ahola@fimnet.fi

Riitta Koivuniemi

riitta.koivuniemi@hus.fi

Jaakko Peltola

jaakko.peltola@saunalahti.fi

Anni Suomalainen

anni.suomalainen@hus.fi

Eeva Moilanen

eeva.moilanen@staff.uta.fi

Mari Hämäläinen

mari.j.hamalainen@staff.uta.fi
Leena Laasonen

laasonen@kolumbus.fi

Jukka H. Meurman

jukka.meurman@helsinki.fi

Marjatta Leirisalo-Repo

ext-marjatta.leirisalo-repo@hus.fi

1 Department of Oral and Maxillofacial Diseases, Helsinki University Hospital, University of Helsinki, Haartmaninkatu 8, PO Box 63, 00014 Helsinki, Finland

2 Department of Bacteriology and Immunology, Helsinki University Hospital, University of Helsinki, Helsinki, Finland

3 Department of Rheumatology, Helsinki University Hospital, University of Helsinki, Helsinki, Finland

4 Department of Radiology, Helsinki University Hospital, University of Helsinki, Helsinki, Finland

5 The Immunopharmacology Research Group, Tampere University Hospital, University of Tampere School of Medicine, Tampere, Finland 


\section{Introduction}

Rheumatoid arthritis (RA) has a worldwide prevalence of $0.5-1 \%$. Due to its chronic and progressive character RA is one of the most important inflammatory rheumatic diseases [1]. The etiology is unknown, however both environmental and genetic factors alone or in different combinations are involved in the onset of RA [2-4].

Infection has been proposed to play a role as a triggering factor for RA [5]. Bacterial biofilm dysbiosis has a significant role in the pathogenesis of oral diseases such as caries and periodontitis [6-8]. RA patients have higher risk for developing infections compared to non-RA subjects [9]. The risk of serious infections increases further in RA patients treated with TNF-inhibitors and/or oral glucocorticoids [9]. Periodontitis associates with RA [10-12]. We have earlier shown that RA patients have more periodontitis, and periodontopathic bacteria in dysbiotic biofilm compared with control population [12].

Based on this background we questioned, (1) if oral inflammation was associated with the development and activity of RA, (2) if oral inflammatory burden was increased in RA patients, and (3) if treatments targeted to suppress the disease activity of RA have an impact on oral health. Therefore, we collected two groups of RA patients, those with early untreated disease (EURA) and those with chronic active RA (CRA) with inadequate response to synthetic disease modifying antirheumatic drugs (DMARDs). As RA patients with active disease often have arthritis in the small joints of hands and wrists, which can lead to impaired hand function and subsequently difficulties in maintaining satisfactory oral hygiene, we further studied if arthritis in hands links to oral health parameters.

\section{Materials and methods}

Eighty-one patients with RA attending the Department of Rheumatology at the Helsinki University Hospital were recruited for rheumatological and oral examinations from September 2005 to May 2014. The patients comprised two groups: (a) 53 patients with DMARD naïve EURA and (b) 28 CRA patients with inadequate response to synthetic DMARDs and were candidates to start treatment with biological DMARDs. We also included 43 population control volunteers matched with the patients for age, gender and community. The controls were recruited using Statistics Finland, the national population database. All participants were Caucasian and from the district of Helsinki University Hospital.
The RA patients were examined by a rheumatologist (ML-R or RK) and by a dentist (KA) at baseline and again at the follow-up [mean $15.9 \pm 6.1$ (SD) months later]. The control volunteers underwent oral examination only once. During the follow-up, two patients moved to another district, six participants interrupted the study for personal reasons; and one EURA patient died between dental and rheumatological examinations. We have previously described the study population and the protocol in more detail [12].

In RA patients, the number of swollen (range 0-66) and tender (0-68) joints were recorded. The patient's global assessment of disease activity (PGA) was recorded on a 100$\mathrm{mm}$ visual analog scale, and 28-joint disease activity score (DAS28) was calculated [13]. The treatment response was evaluated using the European League Against Rheumatism (EULAR) criteria [14]. Physical function was recorded by Health Assessment Questionnaire (HAQ; scale 0-3) [15]. Radiology of hands and feet were taken at baseline and at follow-up. Radiographs were recorded according to the modified Sharp-van der Heijde score (SHS) method [16] by an expert in joint radiology (LL) blinded with regard to the clinical data and treatments. Radiographs were missing at the baseline or after follow-up from 6 patients in EURA group and from 7 patients in CRA group.

Total oral health examinations including panoramic and bite-wing X-rays were performed by the same dentist at the Department of Oral and Maxillofacial Diseases. The total number of teeth taken into account was 28 (third molars were not included based on the WHO recommendation). Decayed missing filled teeth (DMFT), Decayed Missing Filled Surfaces (DMFS) indices and periodontal parameters were recorded according to the WHO recommendations [17]. Periodontal pocket depths (PD) were measured at four tooth sites, and recorded if the values were $\geq 4 \mathrm{~mm}$ [18]. Data from panoramic and bite-wing X-rays were collected by two radiologists (JP and AS) blinded with regard to the clinical examinations. Oral inflammatory burden was evaluated by calculating the Total Dental Index (TDI) according to Mattila et al. [19]. TDI is the sum of scores based on recording caries, periodontitis, periapical and pericoronitis lesions and it may range from 0 to 10 .

Blood samples were collected from RA patients and analyzed for RF, ACPA, C-reactive protein (CRP), erythrocyte sedimentation rate (ESR) and antinuclear antibody (ANA). The upper limit of normal range for RF was $<14 \mathrm{IU} / \mathrm{ml}$ and subjects were considered RF positive or negative based on a single measurement. In addition, plasma samples were collected and centrifuged without delay at $4{ }^{\circ} \mathrm{C}$. Aliquots of $1 \mathrm{ml}$ plasma were transferred to screw-cap polypropylene tubes and frozen at $-80{ }^{\circ} \mathrm{C}$ until later used for analysis of interleukin-6 (IL-6), anti-SSA/SSB (anti-Ro/La) and ribonucleoprotein (RNPAb) antibodies. Blood samples of the 
control subjects were only analyzed for RF, IL-6, CRP and ESR.

IL-6 was determined by enzyme-linked immunoassay (ELISA) using the reagents from eBioscience Inc. (San Diego, CA, USA). The detection limit and interassay coefficient of variation were $0.39 \mathrm{pg} / \mathrm{ml}$ and $4.2 \%$, respectively. Influence of RF and related endogenous antibodies with the IL-6 immunoassay was not found when assayed using commercially available heterophilic blocking agent HeteroBlock (Omega Biologicals, Bozeman, MT, USA) as recommended [20], with concentrations up to $600 \mu \mathrm{g} / \mathrm{ml}$.

Both unstimulated salivary flow rate (USFR) and paraffinwax stimulated flow rate (SSFR) for 5 min were measured according to the routine in our hospital (normal values: unstimulated $>0.1 \mathrm{ml} / \mathrm{min}$, stimulated $>0.7 \mathrm{ml} / \mathrm{min}$ ) [21].

The patients and controls completed a structured questionnaire about their oral and general health habits such as smoking, use of alcohol, tooth brushing, visits to dentist, comorbidities and drugs in use. Also, the subjective symptoms of oral area such as dry mouth were recorded.

All patients and control subjects gave written informed consent to participate in the study.

The study protocol had been approved by the independent review board of the Helsinki and Uusimaa Hospital District (no 240/2004, date 16.6.2004). The study was conducted according to the principles of the Declaration of Helsinki.

\section{Statistical methods}

Normality tests were conducted by Kolmogorov-Smirnov. Continuous variables were compared between groups with Kruskal-Wallis and Mann-Whitney test; the baseline and follow-up values were analyzed by Wilcoxon signed rank test. Correlations for nonparametric data between DMFT, DMFS and DAS28 indices were analyzed by the Spearman rank correlation coefficients. The results are given as medians with minimum and maximum or with 25 th-75th percentiles (IQR). Categorical variables were compared between groups with Chi square test and between baseline and followup values with McNemar's test. The association between DMFS and DAS28 was evaluated by Jonckheere-Terpstra test. Linear regression analysis was performed to establish explanatory factors for DMFT score and unstimulated salivary flow rate. Statistical analyses were performed with SPSS version 22.

\section{Results}

\section{Characteristics of the patients and controls}

The baseline characteristics of the patients and healthy control subjects are given in Table 1. Majority of the RA patients were women. The median ages of the EURA and CRA patients were much the same. In EURA, the median duration of symptoms was 5 months (range 0-29 months, with an outlier who had had symptoms for 12 years without prior diagnosis or treatment), while CRA patients had a long disease history with a median duration of RA 14 years from the diagnosis (range 9 months to 38 years).

\section{Comorbidities}

Comorbidities were common. About half of the patients and controls reported a history of hypertension, thyroid disease, bronchial asthma, depression, cancer or diabetes. Bronchial asthma was more prevalent in EURA patients (19\%) compared to CRA patients $(0 \%)$ and controls $(5 \%)(p=0.046)$.

\section{Rheumatological data}

EURA patients had active disease (Table 2). They had received no DMARD treatment or oral glucocorticoids prior to the baseline rheumatological and dental examinations. During the follow-up the patients received synthetic DMARDs as combination or as monotherapy, systemic lowdose ( $\leq 10 \mathrm{mg}$ prednisolone or equivalent) glucocorticoids, and intra-articular glucocorticoid injections according to the discretion of the treating rheumatologist. At the follow-up examination mean 15.9 (6.1) months later, $17 / 46$ (37.0\%) patients were on monotherapy, 18/46 (39.1\%) on combination of 2 synthetic DMARDs, and 9/46 (19.6\%) on triple therapy. The treatments consisted of methotrexate in 36/46 (78.3\%), sulfasalazine in 18/46 (39.1\%), hydroxychloroquine in $22 / 46(47.8 \%)$ and oral prednisolone in $13 / 46$ (28.3\%) of the patients.

At the follow-up examination, the RA disease activity was significantly decreased in the EURA patients (Table 2). Of the 37 patients examined with complete data after follow-up, $22(48.0 \%)$ were in DAS28 remission (DAS28 $\leq 2.6)$. The EULAR treatment response was good in $23(62.2 \%)$ and moderate in $6(16.2 \%)$ patients, and $8(21.6 \%)$ patients had no response. No significant differences in oral parameters were observed between the EURA patients with respect to EULAR response criteria (data not shown). The response to treatment was also reflected in significantly improved physical function of the patients when assessed with HAQ (Table 2).

The 28 patients with chronic RA had continuing disease activity despite treatment with synthetic DMARDs and glucocorticoids (Table 2). They were candidates for starting treatment with their first biological DMARD. After clinical examinations, biological DMARDs, such as tumor necrosis factor-alpha inhibitors $(22 / 25,88.0 \%)$, anakinra $(1 / 25$, $4.0 \%)$ or rituximab $(2 / 25,8.0 \%)$ were started in 25 patients 
Table 1 Background data for patients with early untreated rheumatoid arthritis (EURA), chronic RA (CRA) and healthy controls at baseline

\begin{tabular}{|c|c|c|c|c|}
\hline & EURA $(N=53)$ & CRA $(N=28)$ & Controls $(N=43)$ & $p$ \\
\hline Women, $N(\%)$ & $45(84.9)$ & $23(82.1)$ & $38(88.4)$ & 0.758 \\
\hline Age, years, median (min-max) & $52(22-78)$ & $54(20-64)$ & $56(30-82)$ & 0.691 \\
\hline RF positive, $N(\%)^{\mathrm{a}}$ & $42(79.2)$ & $18(69.2)$ & $3(8.1)$ & $<0.001$ \\
\hline ACPA positive, $N(\%)^{\mathrm{b}}$ & $37(77.1)$ & $15(78.9)$ & & 0.869 \\
\hline ANA positive, $N(\%)$ & $4(7.5)$ & 0 & & 0.293 \\
\hline SSAAb positive, $N(\%)$ & $4(7.5)$ & $1(3.6)$ & & 0.654 \\
\hline SSBAb positive, $N(\%)$ & $4(7.5)$ & $1(3.6)$ & & 0.654 \\
\hline RNPAb positive, $N(\%)$ & $1(1.9)$ & 0 & & 0.338 \\
\hline Comorbidities, $N(\%)$ & $25(47.2)$ & $16(57.1)$ & $22(51.2)$ & 0.693 \\
\hline Education, $N(\%)^{\mathrm{b}, \mathrm{d}}$ & & & & 0.174 \\
\hline Lower & $20(37.7)$ & $9(36.0)$ & $16(61.5)$ & \\
\hline Secondary & $27(50.9)$ & $12(48.0)$ & $6(23.1)$ & \\
\hline Higher & $6(11.3)$ & $4(16.0)$ & $4(15.4)$ & \\
\hline Smoking, $N(\%)$ & & & & 0.197 \\
\hline Never & $35(66.0)$ & $23(82.1)$ & $36(83.7)$ & \\
\hline Former & $7(13.2)$ & $2(7.1)$ & $1(2.3)$ & \\
\hline Current & $11(20.8)$ & $3(10.7)$ & $6(14.0)$ & \\
\hline Alcohol use, $N(\%)$ & & & & 0.084 \\
\hline Monthly & $28(52.8)$ & $12(42.9)$ & $13(30.2)$ & \\
\hline More often & $25(47.2)$ & $16(57.1)$ & $30(69.8)$ & \\
\hline Tooth brushing at least twice a day, $N(\%)$ & $35(66.0)$ & $22(78.6)$ & $38(88.4)$ & 0.035 \\
\hline Approximal teeth cleaning, $N(\%)$ & $28(52.8)$ & $15(53.6)$ & $34(79.1)$ & 0.018 \\
\hline Self-assessed oral health, $N(\%)^{\mathrm{e}}$ & & & & 0.651 \\
\hline Good & $18(34.6)$ & $10(35.7)$ & $17(39.5)$ & \\
\hline Moderate & $25(48.1)$ & $15(53.6)$ & $23(53.5)$ & \\
\hline Poor or no opinion & $9(17.3)$ & $3(10.7)$ & $3(7.0)$ & \\
\hline
\end{tabular}

The $p$ values were calculated by Kruskal-Wallis test or Chi square test

$A C P A$ anticitrullinated protein antibody, $A N A$ antinuclear antibody, $N$ number of patients, $R A$ rheumatoid arthritis, $S D$ standard deviation, $R F$ rheumatoid factor, $S S A A b$ anti-Sjögren`s-syndrome-related antigen A, $S S B A b$ anti-Sjögren's-syndrome-related antigen $\mathrm{B}, R N P A b$ antibodies for ribonuclear protein

*Modified Sharp van der Heijde method

${ }^{a}$ Data missing from 2 chronic RA patients and 6 controls

${ }^{b}$ Data missing from 9 patients with chronic RA and 5 patients with early RA

${ }^{c}$ Data missing from 3 chronic RA patients

${ }^{\mathrm{d}}$ Data missing from 17 control subjects

${ }^{\mathrm{e}}$ Data missing from 1 early RA patient
(92.6\%); in 23 of them, combined with synthetic DMARD, mostly with methotrexate and prednisolone.

After follow-up, disease activity evaluated by DAS28 was significantly decreased in the CRA patients (Table 2). Of the 23 patients with complete follow-up data, 10 (43.0\%) were in DAS28 remission. The EULAR treatment response was good in 5 patients $(21.7 \%)$, moderate in 9 patients $(39.1 \%)$, and 9 patients $(39.1 \%)$ patients had no response. The improvement was also reflected in HAQ.

The number of patients with tender or swollen joints in fingers or wrists decreased during the study in both RA groups, in patients with early untreated RA from 52 (98.1\%) to $25(56.8 \%)$ and in patients with chronic RA from 25 (89.3\%) to 14 (58.3\%), both $p<0.001$.

Radiological findings increased during the study especially in patients with chronic RA as given in Table 2.

\section{Oral health parameters}

According to the patients' questionnaires, EURA patients brushed their teeth less often than did the CRA patients and controls (Table 1). After follow-up, the number of EURA patients who brushed their teeth at least twice a day had increased statistically significantly from 33 to $37(p=0.044)$. 
Table 2 Disease activity parameters and radiology findings of patients with rheumatoid arthritis at baseline and after follow-up

\begin{tabular}{|c|c|c|c|c|c|c|c|c|}
\hline \multirow[t]{2}{*}{ Parameter, median (IQR) } & \multicolumn{3}{|c|}{ Patients with early untreated RA } & \multicolumn{3}{|c|}{ Patients with chronic RA } & \multirow[t]{2}{*}{$p^{\mathrm{b}}$} & \multirow[t]{2}{*}{$p^{\mathrm{c}}$} \\
\hline & Baseline $(N=53)$ & Follow-up $(N=46)$ & $p^{\mathrm{a}}$ & Baseline $(N=28)$ & Follow-up $(N=26)$ & $p^{\mathrm{a}}$ & & \\
\hline $\begin{array}{l}\text { Tender joint count ( } 68- \\
\text { joint count) }\end{array}$ & $6(3-13)$ & $1(0-6)$ & $<0.001$ & $4(1-8)$ & $1(0-4)$ & 0.055 & 0.081 & 0.830 \\
\hline $\begin{array}{l}\text { Swollen joint count (66- } \\
\text { joint count) }\end{array}$ & $7(4-10)$ & $0(0-2)$ & $<0.001$ & $6(3-11)$ & $1(0-4)$ & $<0.001$ & 0.943 & 0.097 \\
\hline $\begin{array}{l}\text { Erythrocyte sedimenta- } \\
\text { tion rate }(\mathrm{mm} / \mathrm{h})\end{array}$ & $20(11-34)$ & $9(5-16)$ & $<0.001$ & $20(9-46)$ & $15(5-31)$ & 0.026 & 0.850 & 0.206 \\
\hline $\begin{array}{l}\text { Plasma C-reactive pro- } \\
\text { tein }(\mathrm{mg} / \mathrm{l})\end{array}$ & $6(3-14)$ & $3(2-6)$ & 0.001 & $18(5-30)$ & $7(2-19)$ & 0.010 & 0.008 & 0.037 \\
\hline $\begin{array}{l}\text { Plasma interleukin-6 } \\
(\mathrm{pg} / \mathrm{ml})\end{array}$ & $5.3(2.3-17.2)$ & $0.9(0.4-1.7)$ & $<0.001$ & $6.1(2.7-17.5)$ & $2.6(0.8-10.1)$ & 0.727 & 0.478 & 0.002 \\
\hline DAS28 score, 0-10 & $4.0(3.2-4.8)$ & $2.4(1.7-2.9)$ & $<0.001$ & $4.1(3.0-4.9)$ & $3.1(2.0-3.9)$ & 0.003 & 0.974 & 0.097 \\
\hline HAQ score, $0-3$ & $0.5(0.1-0.9)$ & $0.3(0-0.4)$ & $<0.001$ & $0.6(0.4-1.0)$ & $0.3(0-1.0)$ & 0.022 & 0.225 & 0.178 \\
\hline $\begin{array}{l}\text { Patient's global assess- } \\
\text { ment (VAS 0-100 mm) }\end{array}$ & $27(13-48)$ & $14(3-26)$ & $<0.001$ & $43(29-60)$ & $25(10-54)$ & 0.053 & 0.031 & 0.041 \\
\hline \multicolumn{9}{|l|}{ Radiography score* } \\
\hline Erosion & $0(0-2)$ & $0(0-4)$ & $<0.001$ & $17(6-84)$ & $26(5-80)$ & 0.001 & 0.001 & $<0.001$ \\
\hline Narrowing & $0(0-0)$ & $0(0-0)$ & 0.041 & $8(2-56)$ & $13(2-53)$ & 0.002 & $<0.001$ & $<0.001$ \\
\hline Total & $0(0-3)$ & $0(0-4)$ & $<0.001$ & $26(6-136)$ & $36(7-114)$ & 0.001 & $<0.001$ & $<0.001$ \\
\hline $\begin{array}{l}\text { Erosions in hand or foot } \\
\text { radiographs, } N(\%)\end{array}$ & $17(33.3)$ & $24(49.0)$ & $<0.001$ & $22(84.6)$ & $20(90.9)$ & 0.001 & $<0.001$ & $<0.001$ \\
\hline
\end{tabular}

$D A S 28$ Disease Activity Score (28-joint count), HAQ Health Assessment Questionnaire, $N$ number of patients, $R A$ rheumatoid arthritis, VAS visual analog scale

* Modified Sharp van der Heijde method

${ }^{a} p$ value obtained by Wilcoxon test comparing baseline to follow-up

${ }^{\mathrm{b}} p$ value obtained by Kruskal-Wallis test comparing early untreated RA to chronic RA at baseline

${ }^{c} p$ value obtained by Kruskal-Wallis test early untreated RA to chronic RA after follow-up

Table 3 Oral parameters in the study participants

\begin{tabular}{|c|c|c|c|c|c|c|c|c|}
\hline \multirow{2}{*}{$\begin{array}{l}\text { Parameter, median } \\
\text { (IQR) }\end{array}$} & \multicolumn{3}{|c|}{ Patients with early untreated RA } & \multicolumn{3}{|c|}{ Patients with chronic RA } & \multirow[t]{2}{*}{ Controls $(N=43)$} & \multirow[t]{2}{*}{$p^{\mathrm{b}}$} \\
\hline & Baseline $(N=53)$ & Follow-up $(N=47)$ & $p^{\mathrm{a}}$ & Baseline $(N=28)$ & Follow-up $(N=26)$ & $p^{\mathrm{a}}$ & & \\
\hline Number of teeth & $27(23-28)$ & $27(22-28)$ & 0.024 & $27(22-28)$ & $27(22-28)$ & 0.317 & $27(25-28)$ & 0.628 \\
\hline DMFT & $19(12-23)$ & $20(13-24)$ & 0.002 & $19(12-23)$ & $19(12-23)$ & 0.317 & $17(10-21)$ & 0.418 \\
\hline DMFS & $55(27-80)$ & $57(34-83)$ & $<0.001$ & $52(26-79)$ & $56(26-82)$ & 0.008 & $44(18-62)$ & 0.355 \\
\hline TDI & $2(2-3)$ & $2(2-3)$ & 0.349 & $2(1-3)$ & $2(1-3)$ & 0.109 & $1(1-3)$ & 0.045 \\
\hline \multicolumn{9}{|l|}{ Salivary flow $(\mathrm{ml} / 5 \mathrm{~min})$} \\
\hline USFR & $1.0(0.5-1.5)$ & $1.4(0.8-2.2)$ & 0.034 & $1.2(0.7-2.0)$ & $1.7(0.8-2.3)$ & 0.133 & $1.0(0.5-1.2)$ & 0.261 \\
\hline SSFR & $5.0(3.5-7.0)$ & $5.0(3.0-8.6)$ & 0.461 & $5.5(3.8-8.0)$ & $5.0(4.6-8.3)$ & 0.841 & $5.5(4.4-7.0)$ & 0.766 \\
\hline \multicolumn{9}{|l|}{ Hyposalivation, $N(\%)$} \\
\hline $\mathrm{USFR} \leq 0.5 \mathrm{ml} / 5 \mathrm{~min}$ & $16(30.2)$ & $10(21.3)$ & 0.388 & $5(17.9)$ & $3(11.5)$ & 0.688 & $13(30.2)$ & 0.436 \\
\hline $\mathrm{SSFR} \leq 3.5 \mathrm{ml} / 5 \mathrm{~min}$ & $15(30.0)$ & $16(35.6)$ & 0.453 & $6(21.4)$ & $5(19.2)$ & 1.000 & $7(16.3)$ & 0.285 \\
\hline $\begin{array}{l}\text { Self-reported xerosto- } \\
\text { mia, } N(\%)\end{array}$ & $19(35.8)$ & $16(34.8)$ & 1.000 & $11(39.3)$ & $8(30.8)$ & 0.250 & $4(9.3)$ & 0.004 \\
\hline
\end{tabular}

$D M F T$ decayed missing filled teeth, DMFS decayed missing filled surfaces, $N$ number of patient, $R A$ rheumatoid arthritis, $T D I$ total dental index [19], USFR unstimulated salivary flow rate, SSFR stimulated salivary flow rate

${ }^{\mathrm{a}} p$ value comparing baseline and follow-up by Wilcoxon test or McNemar test

${ }^{\mathrm{b}} p$ value obtained by Kruskal-Wallis or Chi square test comparing early untreated and chronic RA patients and Controls at baseline 
No change was observed in the CRA group regarding tooth brushing.

The caries indices DMFT and DMFS of patients in both RA groups did not differ significantly from those of the controls at baseline (Table 3). The DMFS scores slightly increased in both RA groups during the follow-up (Table 3), but no statistically significant difference between EURA and CRA groups was observed in the DMFT and DMFS values after follow-up. The dental inflammation index TDI was similar in both RA groups during the study, but the index was worse in RA patients compared with the control subjects $(p=0.045)$ (Table 3 ). Number of 4-5 mm deep gingival pockets [median (IQR)] was higher in EURA patients [9 (3-18) at baseline and 9 (6-18) after follow-up] vs. CRA patients [5 (3-15) and $4(1-4)]$ and controls [1 (0-4)], $p<0.001$. The number of vertical bone pockets and periapical lesions was also higher in EURA patients through the study, but the results were not statistically significantly different compared to the other study groups (data not shown).

At baseline the RA patients reported subjective xerostomia more often than did the controls (Table 3). The frequency of subjective dry mouth among the EURA or CRA patients did not change statistically significantly from baseline to follow-up. Both USFR and SSFR were on the same level in EURA and CRA groups and in controls at baseline (Table 3). The mean USFR, but not SSFR, improved slightly in the EURA patients during the study.

\section{Serology and oral inflammation}

RF positivity associated with oral inflammation. Including all subjects, those with positive RF $(n=63)$ had higher median (IQR) TDI [2 (2-3)] at baseline compared with RF negative study subjects $(n=53)$ [1 $(1-2), p=0.001]$. Also at baseline the median number of deep pockets (PD $\geq 4 \mathrm{~mm}$ ) was higher in subjects with positive RF $(n=62)$ [9 (3-18)] vs. $3(1-7)$ in RF negative subjects $(n=53, p<0.001)$. The USFR in RF positive RA patients increased from 1.0 ( 0.5 to $1.5)$ to 1.5 (0.8 to 2.2$),(p=0.027)$ during the study.

Four EURA patients and one CRA patient were positive for SSA- and SSB- antibodies, and one EURA patient was positive for RNP antibody. RF was positive in all these six patients and three patients with early untreated RA had positive S-ANA. Three patients with positive SSA/SSB serology reported of dry mouth. Of these patients, two had USFR $\leq 0.5 \mathrm{ml}$. USFR and SSFR values did not differ statistically significantly at baseline between EURA patients with positive and negative SSA/SSB/RNP antibodies. However, after follow-up, the median (IQR) USFR [0.5 (0.2-1.0) vs. $1.5(0.8-2.2), p=0.020]$ and SSFR [2.4 (1.9-3.4) vs. 5.0 (3.3-8.7), $p=0.013$ ] values were significantly lower when comparing the values from these five positive EURA patients to the other EURA patients. DMFT and DMFS indices and
TDI scores were higher at baseline in EURA patients with positive SSA-, SSB- or RNP antibody, respectively, when compared with the rest of the patients $(p=0.039)$ (data not shown).

\section{Disease activity, functional disability, radiological progression and oral health parameters}

Linear regression models were constructed to explore the association of DAS28 and HAQ scores with DMFT index scores and unstimulated salivary flow rates at baseline. The models included both EURA and CRA patients $(n=74)$. In addition to DAS28 and HAQ, the explanatory variables in both models initially included age, gender, RF positivity, and the frequency of tooth brushing. In addition, the DMFT model included the salivary flow rate, while the salivary flow rate model included the number of teeth. Following backward elimination, DAS28 was found to have a positive association with the DMFT index when controlling for age (Table 4). A negative association was found with the unstimulated salivary flow rate when controlling for gender and number of teeth (Table 4). HAQ score was eliminated in both the models.

Decayed missing filled teeth $\left[r_{\mathrm{s}} 0.561(p=0.002)\right]$ and DMFS $\left[r_{\mathrm{s}} 0.581(p=0.001)\right]$ associated with DAS28 at baseline in CRA patients. After follow-up, DAS28 associated positively with DMFT $\left[r_{\mathrm{s}} 0.384(p=0.016)\right]$ and DMFS $\left[r_{\mathrm{s}}\right.$ $0.334(p=0.038)]$ in EURA patients; as well as in CRA patients DMFT $\left[r_{\mathrm{s}} 0.672(p=0.001)\right]$, DMFS $\left[r_{\mathrm{s}} 0.650\right.$ $(p=0.001)]$. Correlation between DAS28 according to low, moderate and high disease activity and DMFS at baseline is

Table 4 Multiple linear regression for Decayed Missing and Filled Teeth (DMFT) index and unstimulated salivary flow rate

\begin{tabular}{lllr}
\hline Dependent & Predictors & Coefficient (95\% CI) & \multicolumn{1}{l}{$p$} \\
\hline DMFT index & Age & $0.346(0.254$ to 0.437$)$ & $<0.001$ \\
& DAS28 score & $1.154(0.240$ to 2.068) & 0.014 \\
Unstimulated & Gender (female) & $-0.173(-0.289$ to & 0.004 \\
$\begin{array}{l}\text { salivary flow } \\
\text { rate }\end{array}$ & & $-0.057)$ & \\
& Number of teeth & & \\
& & $-0.055(-0.106$ to & 0.036 \\
& DAS28 score & $-0.046(-0.080$ to & 0.008 \\
& & $-0.013)$ & \\
\hline
\end{tabular}

The explanatory variables in both models initially included DAS28, HAQ, age, gender, seropositivity, and tooth brushing frequency. In addition, the DMFT model included the salivary flow rate, while the salivary flow rate model included the number of teeth. Statistically significant predictors following backward elimination are shown

CI confidence interval, DMFT decayed missing filled teeth, DAS28 Disease Activity Score, $H A Q$ Health Assessment Questionnaire

${ }^{\text {a }}$ Square root transformation 


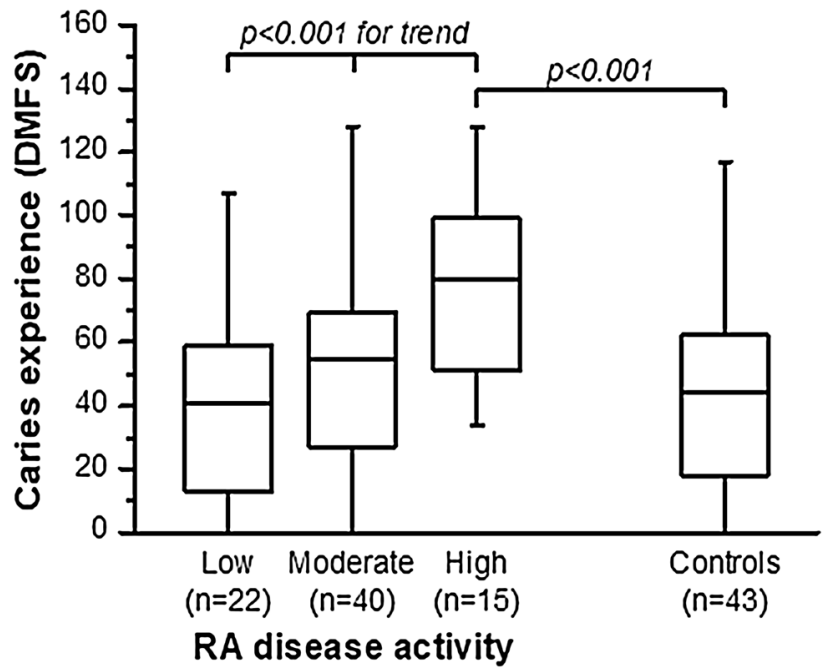

Fig. 1 Caries experience index (DMFS) at baseline in rheumatoid arthritis (RA) patients according to activity of RA

shown in Fig. 1. DMFS had a monotonic increasing association with the activity of RA $(p<0.001)$.

Radiological progression did not correlate statistically significantly with oral health indices at baseline or after follow-up in EURA or CRA group (data not shown).

\section{Discussion}

The main finding in this study was that patients with high activity of RA had increased dental caries index scores (DMFT and DMFS) and increased oral inflammatory burden measured by TDI. Furthermore, study participants with positive RF had higher TDI and increased number of deep periodontal pockets compared to the seronegative participants. Despite the good response to antirheumatic treatment in both RA groups, DMFT and DMFS indices increased during the study. This confirmed our hypothesis that oral inflammation was associated with activity of RA and inflammatory burden was increased in RA patients. Compared with control subjects, TDI also revealed higher inflammation burden (measuring also the data from periodontal condition, periapical lesions and pericoronitis) among patients with early untreated RA before any use of synthetic or biological antirheumatic drugs. Because the majority of patients had arthritis in fingers or wrists, it is likely that poor manual dexterity could contribute to the maintenance of daily oral hygiene. Association between osteoarthritis in hands and disability in maintaining proper oral hygiene has been reported previously [22]. Taking into account the relatively short duration of symptoms (median 5 months) of the patients with early untreated RA, the contribution of deteriorated hand function is probably of minor importance, however. Interestingly, only $50 \%$ from both EURA and CRA patients informed cleaning regularly their approximal tooth spaces. After the institution of treatments with synthetic DMARDs in EURA and biological DMARDs in CRA patients, the number of patients with tender or swollen joints in fingers or wrists decreased in both RA groups and thus the manual dexterity was expected to improve in both these patient groups. Nevertheless, DMFS index scores further increased in both RA groups from baseline to follow-up indicating the constant progression towards poorer oral health.

In general, perceived symptoms of dry mouth are frequent in adult population. Xerostomia is more common in women than in men and its frequency increases with age and associates with the used pharmacotherapy [23]. Oral symptoms and immunological inflammation in salivary glands is a feature of RA. Secondary Sjögren's syndrome is present in about $25 \%$ of RA patients [24]. Saliva protects and lubricates mucosal tissues, prevents microbial accumulation on tooth surfaces and also inhibits bacterial growth. According to an earlier Finnish study, $19.6 \%$ of patients with various inflammatory arthritides, compared with $2.9 \%$ of controls, reported symptoms of dry mouth [25]. The results are in line with the present study, where $36 \%$ of EURA and $40 \%$ of CRA patients at baseline and $10 \%$ of controls, respectively, reported symptoms of dry mouth. The symptom was not alleviated during the follow-up despite the active treatment of RA. Hyposalivation (measured by USFR) was detected in $58 \%$ of patients with early untreated RA and in $28 \%$ of patients with chronic RA with subjective symptoms of dry mouth. Serology test showed positive results for autoantibodies associated with Sjögren's syndrome in six (7.4\%) of our RA patients referring to susceptibility for Sjögren' syndrome. Interestingly, both the unstimulated and stimulated saliva secretion values after follow-up were significantly lower in the autoantibody positive EURA patients than in the rest of the patients. We can only speculate that the patients were prone to develop Sjögren's syndrome. However, because no ocular or oral histopathology tests were conducted, the patients could not be classified to have secondary Sjögren's syndrome [26].

After baseline all the patients received active treatment for RA. Synthetic DMARDs were administered to patients with early untreated RA and biologic DMARDs to patients with chronic RA, with moderate or good treatment response in most of them. Secretion of saliva did not diminish during follow-up, on the contrary, slight improvement was observed. However, hyposalivation (detected in both USFR and SSFR) was present in the minor group of EURA patients with incomplete criteria (positive RF, S-ANA, SSA/ SSB serology, hyposalivation, xerostomia) for secondary Sjögren's syndrome $[24,26]$ after follow-up.

There is only limited information concerning the use of DMARDs with respect to treatment of dry eyes or dry 
mouth in patients with secondary Sjögren's syndrome. Hydroxychloroquine has been commonly used in these patients, but the evidence of this treatment on symptoms of dry eyes is poor [27]. Interestingly, leflunomide, added on methotrexate was reported to decrease tear secretion in RA patients with secondary Sjögren's syndrome, but not in RA patients without preexisting secondary Sjögren's syndrome [28]. Recently, Tsuboi et al. [29] reported that during the treatment with abatacept, a biological drug blocking the T cell co-stimulatory signal, both the tear and saliva volumes increased in RA patients with secondary Sjögren's syndrome.

We did not find any difference in the smoking or use of alcohol between RA patients and controls. This finding is contrary to earlier observations, where smoking in women has shown to be a risk factor of RA [30-32]. Our results might be explained by the low number of smokers in our study, which is in line with the decreasing incidence of smoking in the Finnish population in general [33].

The strength of our study is the homogenous ethnicity of the study population. The patients were followed up prospectively for 1-2 years. Limitation is the fairly small number of patients even though we tried to recruite eligible patients for almost 10 years (2005-2014) and the population covered by the university hospital is 1.5 million. Also, as we did not specifically address the hand function by, e.g., measuring grip strength, the association between arthritic symptoms in the hands and poor oral hygiene remains speculative. Furthermore, due to practical reasons, control subjects were examined only once.

To conclude, our study showed that oral health was worse and oral inflammatory burden higher in RA patients even early at the time of diagnosis compared with population controls without RA. In the RA patients, the activity of RA measured by DAS28 increased with increasing caries indices. Dysbiotic oral environment may be linked to RA already in the entry of the disease. This could be interpreted as a contributory factor to the development of RA or to be associated with impaired manual dexterity due to rheumatic inflammation. Limited maintenance of oral hygiene due to impaired manual dexterity and subsequent poor oral health may, on the other hand, increase the risk of oral infections. This, in turn, could affect disease activity of RA. We emphasize the importance of regular and appropriate dental health care and particularly oral health self-care with individually suitable means in RA patients started from the time of diagnosis of RA. The treatment of oral foci of inflammation is part of the comprehensive treatment of RA focusing to remission of the arthritis.

Author contributions L $\ddot{A}$ is the main investigator in this study which is part of her PhD studies. ML-R and RK recruited and performed the rheumatological examination of the patients, KA carried out dental examinations. JP, AS and LL analyzed the radiological data, and $\mathrm{MH}$ and EM analyzed IL-6 concentrations in plasma. AMH was involved in data analyses and preparing the manuscript, AK was involved in data acquisition, analyses and preparing the manuscript. ML-R and JHM were the PIs of the study design, analyses and in writing and critical evaluation of the manuscript. We did not use any external editing support.

Funding The study was supported by National Graduate School of Musculosceletal Disorders and Biomaterials, by grants from the Helsinki University Hospital Research Funds (EVO-Grants TYH5231, TYH2008232, TYH2011115, TYH2013328, TYH2014225 and TYH2015119), The Medical Society of Finland (Finska Läkaresällskapet) and Liv och Hälsa. The authors thank the patients for participating in this study. Krista Kuuliala PhD, and study nurses Arja Kaarto and Anneli Sinkkonen are acknowledged for their skilled contribution.

\section{Compliance with ethical standards}

Conflict of interest Author Leena Äyräväinen, Author Anna Maria Heikkinen, Author Antti Kuuliala, Author Kirsi Ahola, Author Riitta Koivuniemi, Author Jaakko Peltola, Author Anni Suomalainen, Author Eeva Moilanen, Author Mari Hämäläinen, Author Leena Laasonen, Author Jukka H. Meurman and Author Marjatta LeirisaloRepo declare that they have no conflict of interest.

Ethical standards All procedures performed in studies involving human participants were in accordance with the ethical standards of the institutional and national research committee and with the 1964 Helsinki declaration and its later amendments or comparable ethical standards.

Informed consent Informed consent was obtained from all individual participants included in this study.

\section{References}

1. Gabriel SE, Michaud K (2009) Epidemiological studies in incidence, prevalence, mortality, and comorbidity of the rheumatic diseases. Arthritis Res Ther 11:229. https://doi.org/10.1186/ ar2669

2. Oliver JE, Silman AJ (2006) Risk factors for the development of rheumatoid arthritis. Scand J Rheumatol 35:169-174. https ://doi.org/10.1080/03009740600718080

3. Silman AJ, Pearson JE (2002) Epidemiology and genetics of rheumatoid arthritis. Arthritis Res 4:S265-S272. https://doi. org/10.1186/ar578

4. Alamanos Y, Drosos AA (2005) Epidemiology of adult rheumatoid arthritis. Autoimmun Rev 4:130-136. https://doi. org/10.1016/j.autrev.2004.09.002

5. Carty SM, Snowden N, Silman AJ (2004) Should infection still be considered as the most likely triggering factor for rheumatoid arthritis? Ann Rheum Dis 63:ii46-ii49. https://doi.org/10.1136/ ard.2004.028241

6. Scher JU, Bretz WA, Abramson SB (2014) Periodontal disease and subgingival microbiota as contributors for rheumatoid arthritis pathogenesis: modifiable risk factors? Curr Opin Rheumatol 26:424-429. https://doi.org/10.1097/BOR.0000000000 000076

7. Costerton JW, Stewart PS, Greenberg EP (1999) Bacterial biofilms: a common cause of persistent infections. Science 284:1318 1322. https://doi.org/10.1126/science.284.5418.1318 
8. Marsh PD (2010) Microbiology of dental plaque biofilms and their role in oral health and caries. Dent Clin N Am 54:441-454. https ://doi.org/10.1016/j.cden.2010.03.002

9. Singh JA (2016) Infections With biologics in rheumatoid arthritis and related conditions: a scoping review of serious or hospitalized infections in observational studies. Curr Rheumatol Rep 18:61. https://doi.org/10.1007/s11926-016-0609-5

10. Detert J, Pischon N, Burmester GR, Buttgereit F (2010) The association between rheumatoid arthritis and periodontal disease. Arthritis Res Ther 12:218. https://doi.org/10.1186/ar3106

11. Araújo VMA, Melo IM, Lima V (2015) Relationship between periodontitis and rheumatoid arthritis: review of the literature. Mediators Inflamm 2015:259074. https://doi.org/10.1155/2015/259074

12. Äyräväinen L, Leirisalo-Repo M, Kuuliala A, Ahola K, Koivuniemi R, Meurman JH, Heikkinen AM (2017) Periodontitis in early and chronic rheumatoid arthritis: a prospective follow-up study in Finnish population. BMJ Open 7:e011916. https://doi. org/10.1136/bmjopen-2016-011916

13. Prevoo MLL, vant't Hof MA, Kuper HH, van Leeuwen MA, van de Putte LB, van Riel PL (1995) Modified disease activity scores that include twenty-eight-joint counts. Development and validation in a prospective longitudinal study of patients with rheumatoid arthritis. Arthritis Rheum 38:44-48

14. van Gestel AM, Prevoo MLL, vant't Hof MA, van Rijswijk MH, van de Putte LB, van Riel PL (1996) Development and validation of the European League Against Rheumatism response criteria for rheumatoid arthritis. Comparison with the preliminary American College of Rheumatology and the World Health Organization/ International League Against Rheumatism Criteria. Arthritis Rheum 39:34-40

15. Hakala M, Nieminen P, Manelius J (1994) Joint impairment is strongly correlated with disability measured by self-report questionnaires. Functional status assessment of individuals with rheumatoid arthritis in a population based series. J Rheumatol 21:64-69

16. van der Heijde DM, van Leeuwen MA, van Riel PL, Koster AM, van't Hof MA, van Rijswijk MH, van de Putte LB (1992) Biannual radiographic assessments of hands and feet in a three-year prospective follow up of patients with early rheumatoid arthritis. Arthritis Rheum 35:26-34

17. World Health Organisation (1997) Oral health survey basic methods, 5 th edn. WHO, Geneva

18. Tomar SL, Asma S (2000) Smoking-attributable periodontitis in the United States: findings from NHANES III. National Health and Nutrition Examination Survey. J Periodontol 71:743-751. https://doi.org/10.1902/jop.2000.71.5.743

19. Mattila KJ, Nieminen MS, Valtonen VV, Rasi VP, Kesäniemi YA, Syrjälä SL, Jungell PS, Isoluoma M, Hietaniemi K, Jokinen MJ (1989) Association between dental health and acute myocardial infarction. BMJ 298:779-781

20. Todd DJ, Knowlton N, Amato M, Frank MB, Schur PH, Izmailova ES, Roubenoff R, Shadick NA, Weinblatt ME, Centola M, Lee DM (2011) Erroneous augmentation of multiplex assay measurements in patients with rheumatoid arthritis due to heterophilic binding by serum rheumatoid factor. Arthritis Rheum 63:894-903. https://doi.org/10.1002/art.30213

21. Villa A, Connell CL, Abati S (2015) Diagnosis and management of xerostomia and hyposalivation. Ther Clin Risk Manag 11:4551. https://doi.org/10.2147/TCRM.S76282
22. Kelsey JL, Lamster IB (2008) Influence of musculoskeletal conditions on oral health among older adults. Am J Public Health 98:1177-1183. https://doi.org/10.2105/AJPH.2007.129429

23. Nederfors T, Isaksson R, Mörnstad H, Dahlöf C (1997) Prevalence of perceived symptoms of dry mouth in an adult Swedish population-relation to age, sex and pharmacotherapy. Community Dent Oral Epidemiol 25:211-216

24. Kassan SS, Moutsopoulos HM (2004) Clinical manifestations and early diagnosis of Sjögren syndrome. Arch Intern Med 164:12751284. https://doi.org/10.1001/archinte.164.12.1275

25. Ahola K, Saarinen A, Kuuliala A, Leirisalo-Repo M, Murtomaa H, Meurman JH (2015) Impact of rheumatic diseases on oral health and quality of life. Oral Dis 21:342-348. https://doi. org/10.1111/odi.12285

26. Vitali C, Bombardieri S, Jonsson R, Moutsopoulos HM, Alexander EL, Carsons SE, Daniels TE, Fox PC, Fox RI, Kassan SS, Pillemer SR, Talal N, Weisman MH, European Study Group on Classification Criteria for Sjögren's Syndrome (2002) Classification criteria for Sjögren's syndrome: a revised version of the European criteria proposed by the American-European Consensus Group. Ann Rheum Dis 61:554-558. https://doi.org/10.1136/ ard.61.6.554

27. Akpek EK, Lindsley KB, Adyanthaya RS, Swamy R, Baer AN, McDonnell PJ (2011) Treatment of Sjögren's syndrome-associated dry eye an evidence-based review. Ophthalmology 118:12421252. https://doi.org/10.1016/j.ophtha.2010.12.016

28. Shahin AA, El-Agha S, El-Azkalany GS (2014) The effect of leflunomide on the eye dryness in secondary Sjögren's syndrome associated with rheumatoid arthritis and in rheumatoid arthritis patients. Clin Rheumatol 33:925-930. https://doi.org/10.1007/ s10067-014-2548-8

29. Tsuboi H, Matsumoto I, Hagiwara S, Hirota T, Takahashi H, Ebe H, Yokosawa M, Yagishita M, Takahashi H, Kurata I, Ohyama A, Honda F, Asashima H, Miki H, Umeda N, Kondo Y, Hirata S, Saito K, Tanaka Y, Horai Y, Nakamura H, Kawakami A, Sumida T (2016) Effectiveness of abatacept for patients with Sjögren's syndrome associated with rheumatoid arthritis. An open label, multicenter, one-year, prospective study: ROSE (Rheumatoid Arthritis with Orencia Trial toward Sjögren's syndrome Endocrinopathy) trial. Mod Rheumatol 25:187-193. https://doi.org/10.3109/14397 595.2016.1158773

30. Hazes JM, Dijkmans BA, Vandenbroucke JP, de Vries RR, Cats A (1990) Lifestyle and the risk of rheumatoid arthritis: cigarette smoking and alcohol consumption. Ann Rheum Dis 49:980-982

31. Krishnan E, Sokka T, Hannonen P (2003) Smoking-gender interaction and risk for rheumatoid arthritis. Arthritis Res Ther 5:158 162. https://doi.org/10.1186/ar750

32. Johannsen A, Susin C, Gustafsson A (2014) Smoking and inflammation: evidence for a synergistic role in chronic disease. Periodontol 2000 64:111-126. https://doi.org/10.111 1/j.1600-0757.2012.00456.x

33. Borodulin K, Vartiainen E, Peltonen M, Jousilahti P, Juolevi A, Laatikainen T, Männistö S, Salomaa V, Sundvall J, Puska P (2015) Forty-year trends in cardiovascular risk factors in Finland. Eur J Public Health 25:539-546. https://doi.org/10.1093/eurpub/cku17 